\title{
Separating diffraction from scattering: the million-dollar challenge
}

\author{
Philip Laven \\ 9 Russells Crescent, Horley, RH6 7DJ, United Kingdom \\ philip@philiplaven.com
}

\begin{abstract}
Craig Bohren has offered a million-dollar prize to anyone who can devise a detector that accepts scattered light but rejects diffracted light. This challenge was examined from a theoretical perspective by considering the scattering of red light by a spherical droplet of water with diameter $20 \mu \mathrm{m}$. Illumination of the droplet by short pulses (e.g. a duration of 5 fs) could allow a detector to distinguish between light scattered by various mechanisms, such as diffraction, transmission, reflections and surface waves. Although such techniques would not satisfy the precise terms of the challenge, the time domain approach can deliver remarkable insights into the details of the scattering processes
\end{abstract}

Keywords: scattering, diffraction, surface waves, Lorenz-Mie theory, Debye series, femtosecond pulses, impulse response.

\section{INTRODUCTION}

Craig Bohren recently sent me an e-mail which included the sentence "For many years I have offered a million-dollar prize to anyone who can devise a detector that accepts scattered light but rejects diffracted light." On the basis that it is possible to win a lottery (even if it is highly unlikely), Craig's e-mail made me wonder if his million-dollar challenge was as impossible as it seemed. I started by assuming that the formal rules for claiming the prize were defined by the statement on page 126 of the book Fundamentals of Atmospheric Radiation by Bohren and Clothiaux [1], as reproduced below:

"For many years we have offered a million-dollar prize to anyone who can devise a detector that distinguishes between scattered and diffracted waves, accepting the one but rejecting the other."

An immediate issue is that, in my opinion, "scattering" is a generic term that includes all manner of scattering mechanisms, such as diffraction, reflection, transmission, surface waves, etc. Hence, using my own definition, the challenge is impossible. On the other hand, as the challenge is about physics (rather than about semantics), I assumed that the task actually involves separating the diffracted components from those components that have been scattered by any mechanism other than diffraction.

This paper explores the problems set by the challenge and suggests a possible partial solution.

All of the graphs and diagrams in this paper have been generated using the MiePlot computer program which is freely available from http://www.philiplaven.com/MiePlot.htm. This program was originally developed to provide a user-friendly interface to Bohren \& Huffman's code known as BHMIE [2] for the specific purpose of investigating scattering of light by droplets of water in the atmosphere. Hence, this paper uses the example of scattering of red light by a spherical water droplet of $20 \mu \mathrm{m}$ diameter. 


\section{ANALYZING THE PROBLEM}

\subsection{Lorenz-Mie theory and the Debye series}

To investigate this problem, I decided to consider scattering by a spherical droplet of water of diameter $d=20 \mu \mathrm{m}$. The results of calculations based on Lorenz-Mie theory (LMT) for the scattering of red light $(\lambda=650 \mathrm{~nm})$ are shown in Fig. 1 - which shows that the maximum intensity of scattering occurs when the scattering angle $\theta=0^{\circ}$ (corresponding to forward scattering).

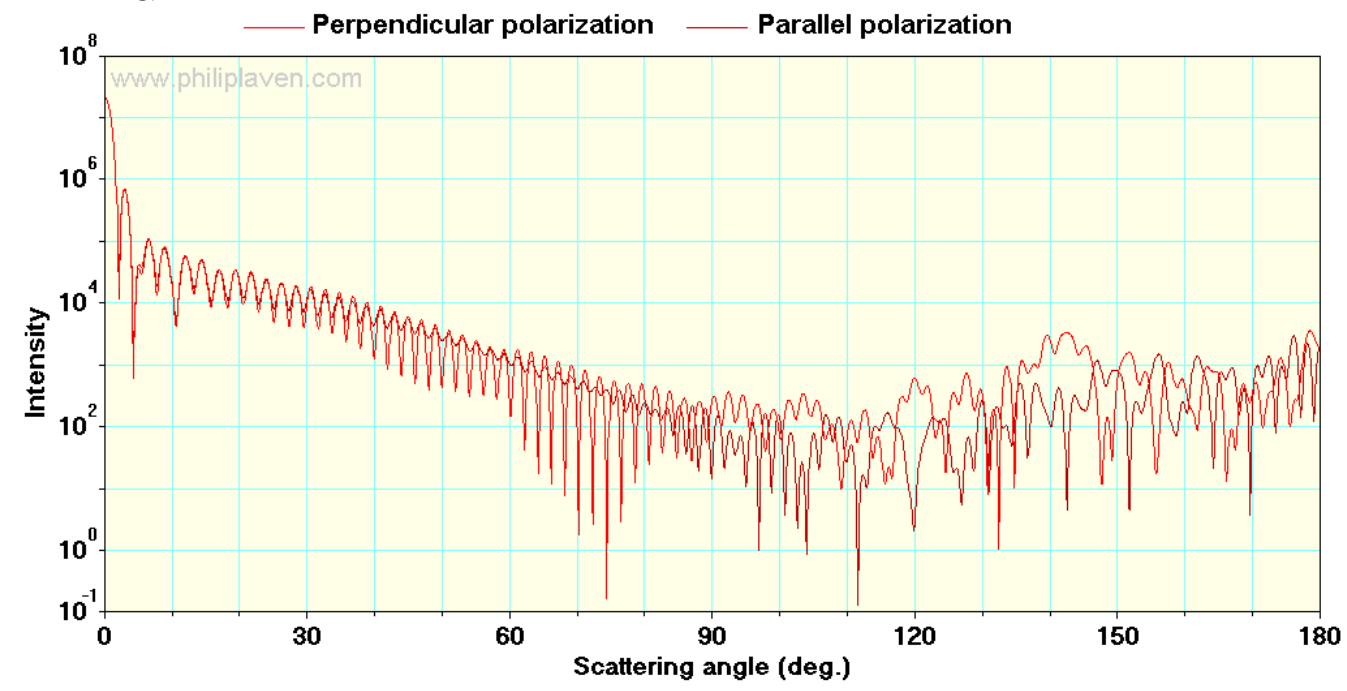

Fig. 1. LMT calculations for scattering of red light $(\lambda=650 \mathrm{~nm})$ by a spherical homogeneous drop of water of diameter $d=20 \mu \mathrm{m}$ (refractive index of sphere $n_{l}=1.3326+\mathrm{i} 1.67 \mathrm{E}-08$ ) in a medium with refractive index $n_{0}=1$.

The complicated ripples shown in Fig. 1 indicate that scattering by a sphere is not a simple process. However, as an aid to understanding Fig. 1, it is useful to introduce the Debye series $[3,4,5]-$ which is essentially a reformulation of LMT separating the contributions made by different scattering mechanisms of order $p$ (see Fig. 2), where:

- $\quad p=0$ corresponds to external reflection plus diffraction;

- $p=1$ corresponds to direct transmission through the sphere;

- $\quad p=2$ corresponds to one internal reflection;

- $p=3$ corresponds to two internal reflections; and so on ...

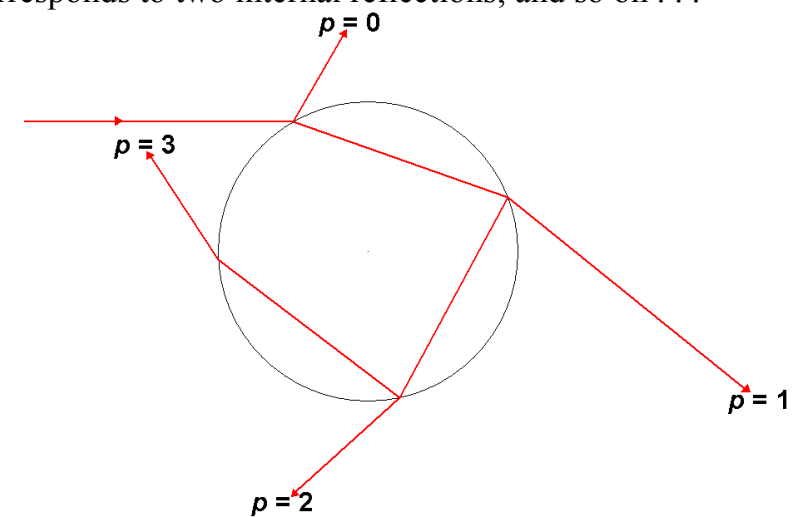

Fig. 2. Geometrical ray paths corresponding to scattering orders $p=0,1,2$ and 3 . 
Comparing the results of Debye series calculations in Fig. 3 with the LMT calculations in Fig. 1, it will be noted that $p=0$ scattering (i.e. external reflection plus diffraction) is responsible for the forward scattering peak as $\theta \rightarrow 0^{\circ}$. Similarly, $p=2$ scattering (one internal reflection) is responsible for the primary rainbow at $\theta \approx 142^{\circ}$, whilst $p=3$ scattering (two internal reflections) is responsible for the secondary rainbow at $\theta \approx 124^{\circ}$. Note that the primary and secondary rainbows are not well-defined in terms of scattering angle $\theta$ - even for scattering of monochromatic light. When sunlight is scattered by water droplets with $d<40$ $\mu \mathrm{m}$, the resulting rainbows are almost white - and are known as fogbows or cloudbows. Scattering of sunlight by larger drops of water, such as $d>200 \mu \mathrm{m}$, produces the familiar colored primary and secondary rainbows (with red arcs at $\theta \approx 138^{\circ}$ and $\theta \approx 129^{\circ}$ respectively).

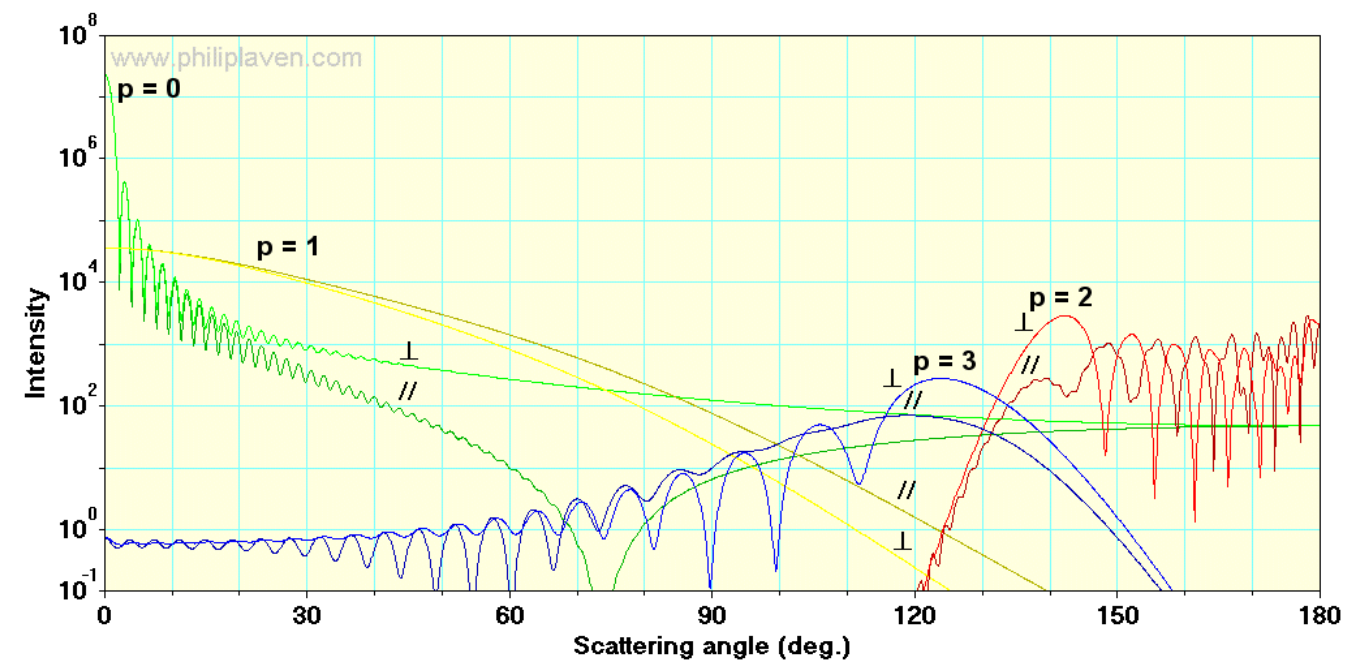

Fig. 3. As Fig. 1, but showing the results of calculations using the Debye series for selected values of $p$. The curves for perpendicular polarization are marked with the symbol $\perp$, whilst the curves for parallel polarization are marked with $/ /$.

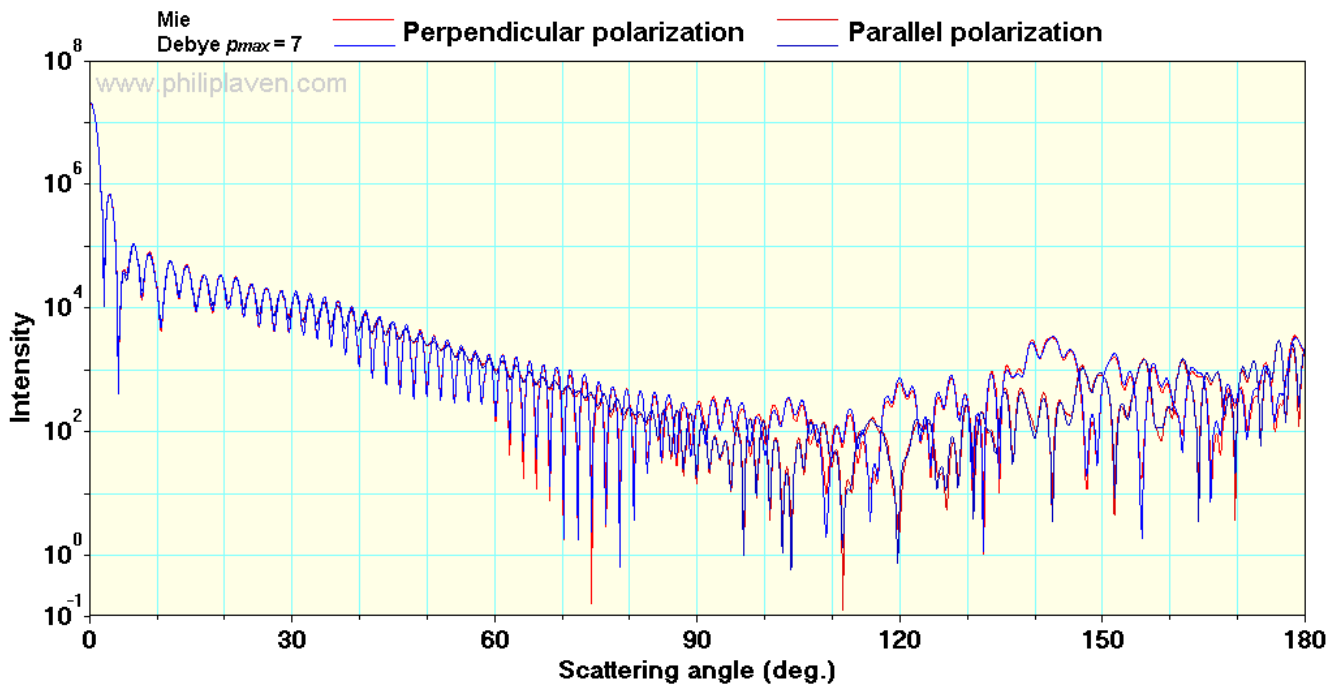

Fig. 4. As Fig. 1: the red lines show the results of LMT calculations, whilst the blue lines show the vector sum of the Debye series results for $p=0$ through $p=7$ (i.e. $p_{\max }=7$ ). 
It should be noted that the Debye series is not an approximation. The sum of the Debye series for all integer values of $p$ from $p=0$ to $p=\infty$ gives the same result as calculations based on LMT. In practice, this vector sum can generally be safely truncated at a much lower value of $p$, as shown in Fig. 4 where the blue curves representing the combined intensity of the Debye results for $p=0$ through $p=7$ have been superimposed on the red curves representing the LMT results. In this case, truncating the sum at $p_{\max }=7$ gives results very close to the LMT results, whilst increasing $p_{\max }$ to 12 gives results that are essentially identical to the LMT results. The Appendix gives further information about the Debye series.

\subsection{Fraunhofer approximation and ray-tracing}

The separation provided by the Debye series might be part of the solution to the challenge, except for the awkward fact that the Debye $p=0$ term combines diffraction with reflection from the exterior of the sphere. As the challenge involves "diffraction", it is important to be clear about the meaning of diffraction. In the case of scattering by a spherical particle, the diffraction pattern is typically defined by the Fraunhofer approximation $[2,6,7]$ :

$$
\mathrm{S}(\theta)=x^{2}\left[\frac{1+\cos (\theta)}{2}\right]\left[\frac{\mathrm{J}_{1}(x \sin (\theta))}{x \sin (\theta)}\right]
$$

where:

- $S(\theta)$ is the amplitude of the scattered field at scattering angle $\theta$;

- $\mathrm{J}_{1}$ is the first-order Bessel function;

- $x=2 \pi r / \lambda$ where $r$ is the radius of the scattering sphere and $\lambda$ is the wavelength of the incident light.

Such equations are frequently attributed to Fraunhofer, but Craig Bohren has pointed out in a private communication that, without diminishing the importance of Fraunhofer's pioneering work in experimental optics, there is no evidence to suggest that Fraunhofer developed any theoretical treatment of diffraction. Consequently, he has suggested that "Fresnel-Fraunhofer-Airy-Schwerd" might be a more appropriate designation.

The essence of the challenge is shown in Fig. 5 which plots results of calculations for three distinct scattering mechanisms:

- diffraction (calculated using Fraunhofer's approximation, Eq. (1));

- $p=0$ reflection from the exterior of the sphere (calculated using ray-tracing and Fresnel's equations);

- $\quad p=1$ transmission through the sphere (calculated using ray-tracing and Fresnel's equations).

Fig. 6 compares the vector sum of the Fraunhofer approximation and the $p=0$ reflection term shown in Fig. 4 (taking phase differences into account) with the results of Debye $p=0$ calculations (which combine diffraction and reflection from the exterior of the sphere). Although the two curves in Fig. 6 show good agreement between for the first few maxima and minima (i.e. for $\theta<10^{\circ}$ ), the significant discrepancies at higher values of $\theta$ (particularly the larger amplitudes of the ripple on the blue curve) suggest that the Fraunhofer approximation over-estimates the intensity of the diffracted component beyond the first few maxima.

Figs. 3 and 5 indicate that diffraction is the dominant mechanism causing forward scattering (e.g. $0^{\circ}<\theta<1^{\circ}$ ), whilst transmission through the sphere $(p=1)$ is dominant when $10^{\circ}<\theta<30^{\circ}$. Unfortunately, vague phrases such as "dominant" are not sufficient to satisfy any challenge which demands complete "separation" of scattering mechanisms. 


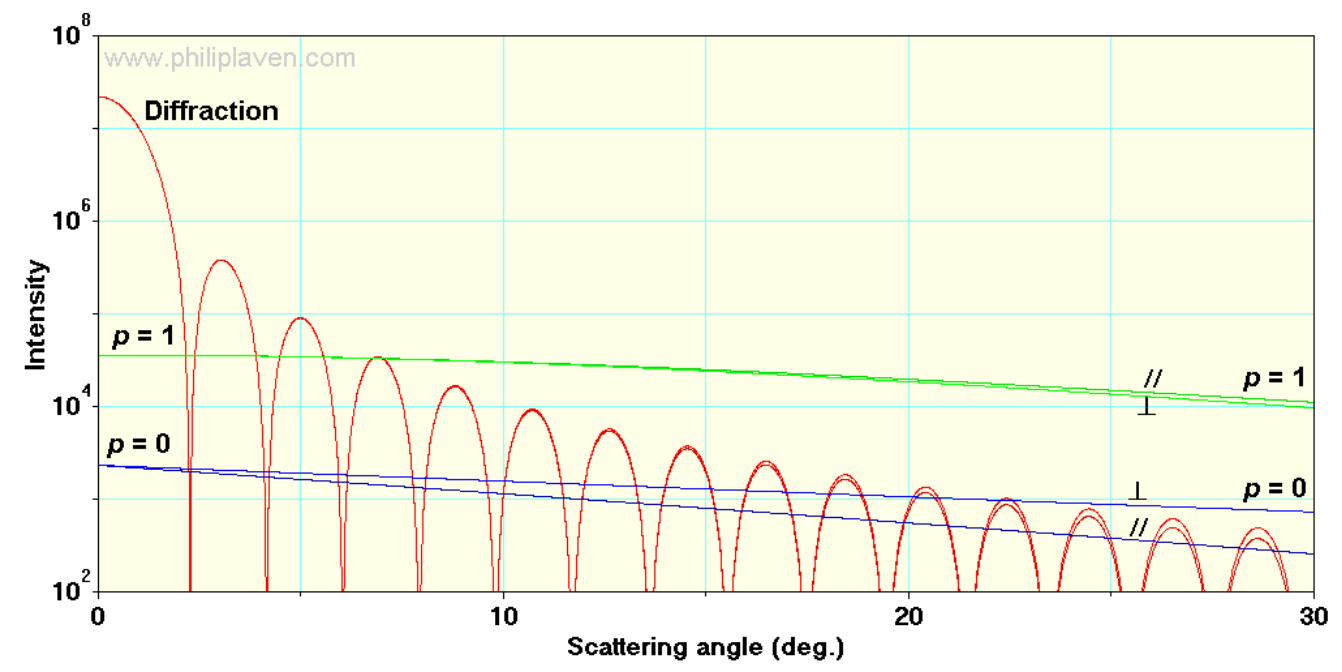

Fig. 5. As in Fig. 1 (limited to $0^{\circ}<\theta<30^{\circ}$ ), but using the Fraunhofer approximation for diffraction shown in Eq. (1), ray-tracing for reflection from the exterior of the sphere $(p=0)$ and ray-tracing for transmission through the sphere $(p=1)$. The curves for perpendicular polarization are marked with the symbol $\perp$, whilst the curves for parallel polarization are marked with the symbol //.

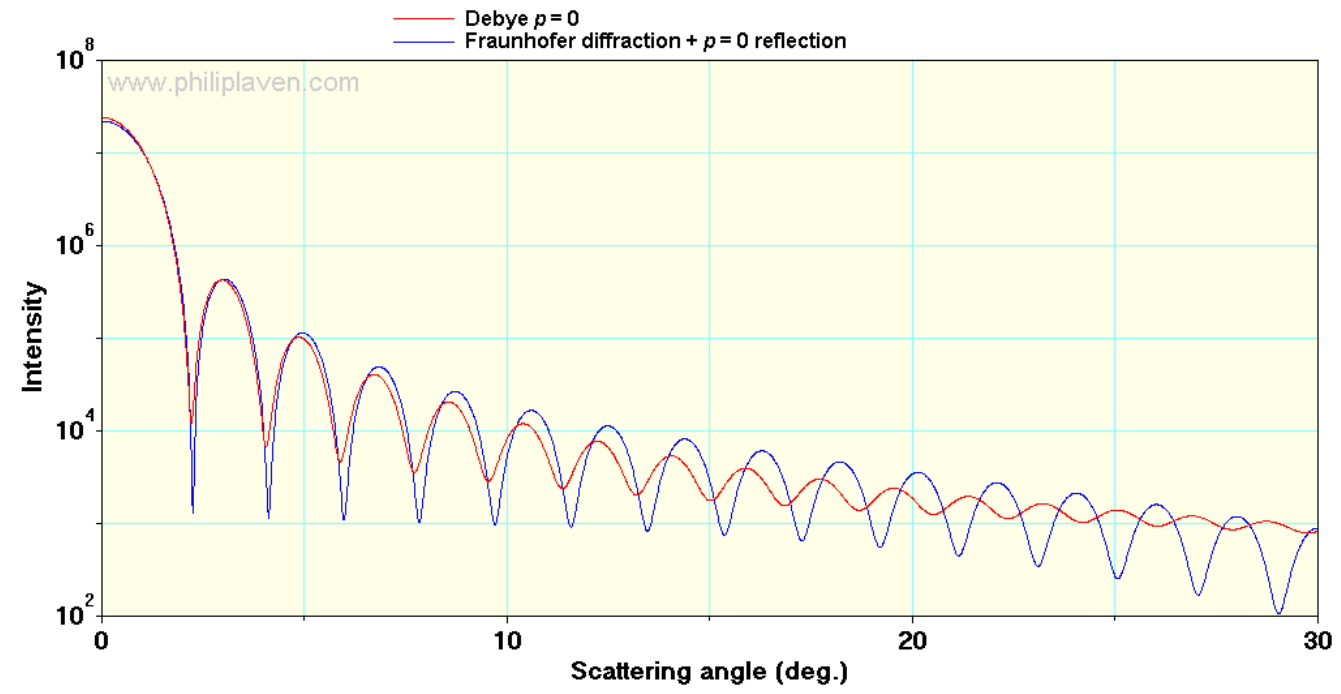

Fig. 6. Scattering for the same conditions as in Fig. 5, but comparing the results of calculations based on the Fraunhofer approximation for diffraction and ray-tracing for reflection from the exterior of the sphere $(p=0)$ with the results of Debye $p=0$ calculations (perpendicular polarization only).

Although the above results show that it is possible identify the contributions caused by different scattering mechanisms, Bohren's challenge would not be satisfied by a mathematical construct. It demands a measurement technique that will reveal the intricate details of the scattering processes, but there seems to be little hope of success because scattering at a given angle $\theta$ is due to a combination of scattering mechanisms.

Despite this apparently insuperable problem, is it possible to devise a measurement technique that could, in some way, replicate the theoretical results of the Debye series? Ideally, the intensity of the various scattering mechanisms should be retrieved directly from 
measurements - even if the intensity at a given angle $\theta$ for a specific scattering mechanism is much weaker than the dominant scattering mechanism. Taking the example of Fig. 5, this implies the need for direct measurements of ALL three scattering mechanisms for $0<\theta<30^{\circ}$. This objective is potentially much more difficult than Craig Bohren's challenge of separating the diffracted and non-diffracted components.

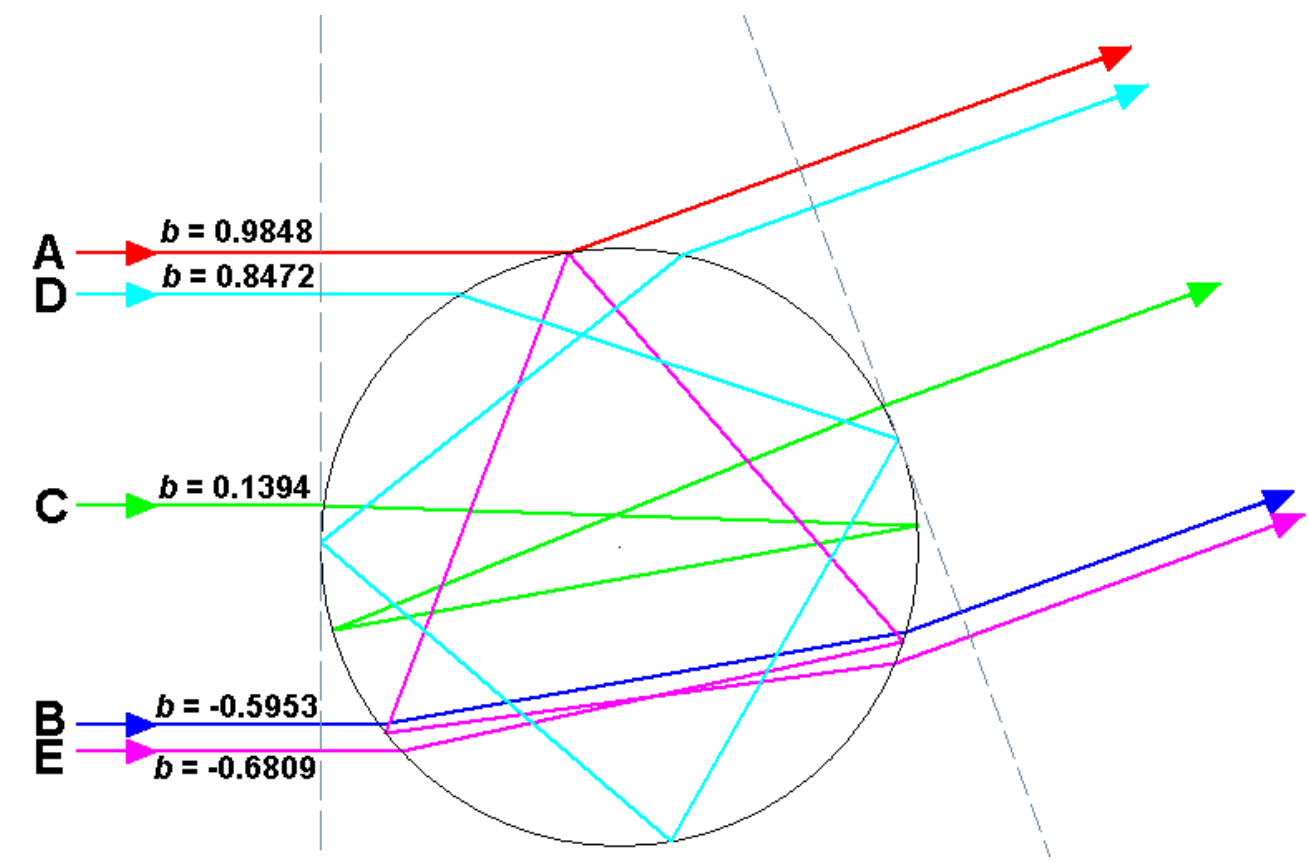

Fig. 7. Geometric rays that result in counter-clockwise deviation of $20^{\circ}$ for a sphere with refractive index $n_{l}=1.3326$ in a medium with refractive index $n_{0}=1$ for $p=0$ through $p=4$.

A beam of light can be considered as a set of parallel rays - as shown in Fig. 7 which selects a few rays from the incident beam of light that are deviated by an angle of $20^{\circ}$ in a counter-clockwise direction. Table 1 shows the characteristics of the propagation paths for each of the rays. The impact parameter $b$ measures the perpendicular distance of the path of an incident ray from the center of the scattering sphere: for example, an impact parameter $b=$ 0 corresponds to an incident ray aimed at the center of the sphere, whilst an impact parameter $b= \pm 1$ corresponds to an incident ray that is tangential to the top or bottom of sphere. Each of the rays shown in Fig. 7 suffers a delay dependent on its propagation path through the sphere. Table 1 shows the calculated time $\tau$ in femtoseconds $\left(1 \mathrm{fs}=10^{-15} \mathrm{~s}\right)$ taken by each ray to travel between the dashed reference lines on the left and right of the sphere (for a spherical droplet of water with diameter $d=20 \mu \mathrm{m}$ ).

\begin{tabular}{|c|c|c|c|}
\hline Ray & $\boldsymbol{p}$ & Impact parameter $\boldsymbol{b}$ & Time delay $\boldsymbol{\tau}(\mathbf{f s})$ \\
\hline $\mathrm{A}$ & 0 & 0.9848 & 55.1 \\
\hline $\mathrm{B}$ & 1 & -0.5953 & 92.6 \\
\hline $\mathrm{C}$ & 3 & 0.1394 & 265.7 \\
\hline $\mathrm{D}$ & 4 & 0.8472 & 305.5 \\
\hline $\mathrm{E}$ & 4 & -0.6809 & 323.3 \\
\hline
\end{tabular}

Table 1 Propagation parameters for each of the rays shown in Fig. 7 for a water droplet with diameter $d=20 \mu \mathrm{m}$. 


\subsection{Calculations in the time domain}

The values of $\tau$ listed on Table 1 suggest a way of identifying the various contribution to scattering at $\theta=20^{\circ}$. Instead of illuminating a sphere with a light source of constant intensity, the sphere could be illuminated by an extremely short pulse of light so that a detector at $\theta=20^{\circ}$ could measure the resulting impulse response.

Such measurements would not necessarily be easy to perform, but I have recently extended the MiePlot computer program to calculate the impulse response of a sphere (based on the work of Bech \& Leder $[8,9])$. This program first performs a Fast Fourier Transform (FFT) on the pulse shape in the time domain to determine the spectrum of the pulse, which is then multiplied by the results of scattering calculations for a range of scattering angles $\theta$ at a number of discrete wavelengths across the bandwidth of the pulse. The results for a given value of $\theta$ as a function of wavelength are then subjected to another FFT so as to produce the time domain impulse response for that value of $\theta$.

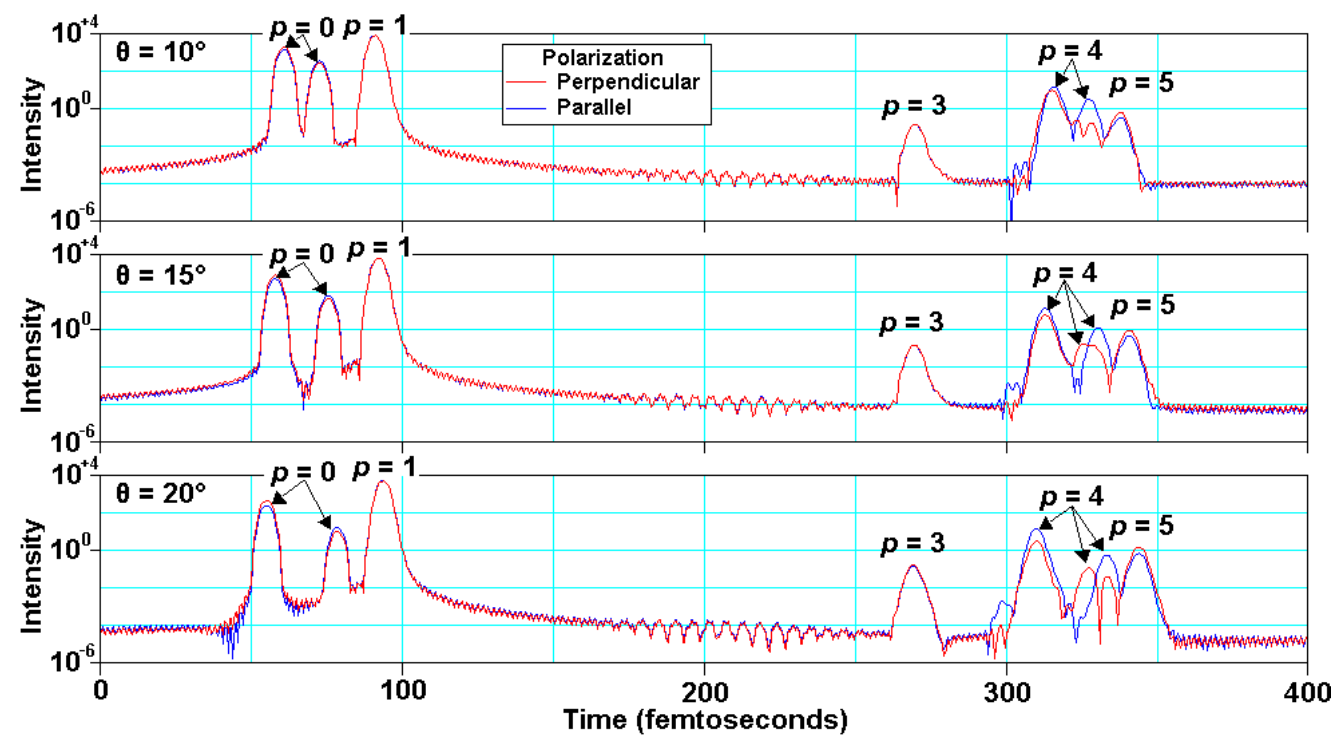

Fig. 8 Impulse response of a spherical droplet of water $(d=20 \mu \mathrm{m})$ for a 5 fs pulse of red light for scattering angles $\theta=10^{\circ}, 15^{\circ}$ and $20^{\circ}$ (taking account of dispersion).

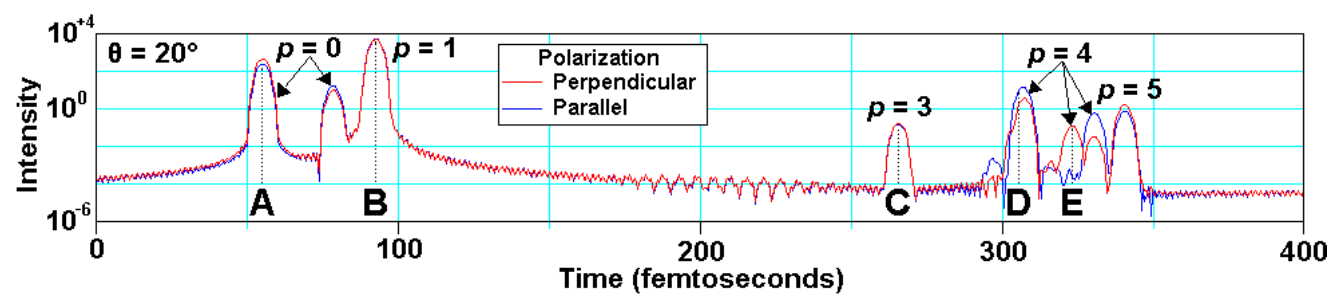

Fig. 9 Impulse response of a spherical droplet of water $(d=20 \mu \mathrm{m})$ for a 5 fs pulse of red light for scattering angle $\theta=20^{\circ}$ (assuming fixed refractive index $n_{l}=1.3326+\mathrm{i} 1.67 \mathrm{E}-08$ ). 
The graphs in Fig. 8 show the calculated impulse responses using LMT for scattering angles $\theta=10^{\circ}, 15^{\circ}$ and $20^{\circ}$ for the following conditions:

- Nominal wavelength $\lambda=650 \mathrm{~nm}$

- Pulse duration: $5 \mathrm{fs}$ (half-amplitude duration, raised-cosine pulse shape)

- Pulse bandwidth: $564 \mathrm{~nm}-767 \mathrm{~nm}(-3 \mathrm{~dB}) ; 404 \mathrm{~nm}-1664 \mathrm{~nm}(-40 \mathrm{~dB})$

- Sphere diameter: $d=20 \mu \mathrm{m}$

- Refractive index of sphere: $n_{l}=1.3326+\mathrm{i} 1.67 \mathrm{E}-08$ at nominal wavelength $\lambda=650$ $\mathrm{nm}$ (N.B. The real part of the refractive index of water is 1.344 at $404 \mathrm{~nm}$ and 1.313 at $1664 \mathrm{~nm}$, whilst the imaginary part is negligible for the current purposes.)

- Refractive index of medium: $n_{0}=1$

The various pulses shown in Fig. 8 can be identified by separate calculations using the Debye series. In particular, each graph shows two pulses labeled " $p=0$ ". As $\theta$ is increased from $10^{\circ}$ to $20^{\circ}$, the first pulse moves from $\tau \approx 61$ fs to $\tau \approx 55 \mathrm{fs}$, whereas the second pulse moves from $\tau \approx 72$ fs to $\tau \approx 78$ fs. By default, the MiePlot program takes account of variations of refractive index of water across the bandwidth of the pulse, but to facilitate comparisons with the results in Table 1, Fig. 9 shows the impulse response for $\theta=20^{\circ}$ assuming a constant value of refractive index (whereas Fig. 8 takes account of dispersion). Note that the letters A to $\mathrm{E}$ in Fig. 9 correspond to the geometric rays shown in Fig. 7. Examination of the results for $\theta=20^{\circ}$ in Fig. 8 and 9 shows that the pulses for $p>0$ in Fig. 8 have been broadened and shifted by dispersion.

The close agreement between the values of $\tau$ given by the various independent methods of calculation (i.e. LMT, Debye and ray-tracing) gives considerable confidence in the results. However, it is also important to recognize that some of the scattered pulses shown for $\theta=20^{\circ}$ in Fig. 9 were not predicted by the ray-tracing exercise in Fig. 7 - for example, there is an extra $p=0$ pulse at $\tau \approx 78$ fs and an extra $p=4$ pulse at $\tau \approx 330 \mathrm{fs}$.

What causes these "non-geometrical" pulses? The fact that the $p=4$ pulse at $\tau \approx 330 \mathrm{fs}$ is dominated by parallel polarization suggests the involvement of surface waves - which are typically generated by rays with impact parameter $b=1$ or $b=-1$. Fig. 10(a) shows a ray with impact parameter $b=1$ suffering 3 internal reflections before generating a surface wave which travels $9^{\circ}$ clockwise along the circumference of the sphere, resulting in scattering at $\theta=$ $20^{\circ}$ with $\tau=306.8 \mathrm{fs}$. As this delay is extremely close to that of geometrical ray $\mathrm{D}(\tau=305.5$ $\mathrm{fs}$ ), it is not possible to distinguish between these two propagation paths with a pulse of $5 \mathrm{fs}$ duration. However, as shown by Fig. 10(b), there is another $p=4$ path involving surface waves: in this case, a ray with impact parameter $b=-1$ suffers 3 internal reflections before generating a surface wave which travels $49^{\circ}$ counterclockwise along the circumference of the sphere, resulting in scattering at $\theta=20^{\circ}$ with $\tau=330.1 \mathrm{fs}$ - which agrees well with LMT results in Fig. 9. The ray paths shown in Figs. 10(a) and (b) are not symmetrical because the lengths of arc travelled by the surface waves are different. The path shown in Fig. 10(b) is $40^{\circ}$ of arc longer than the path shown in Fig. 10(a). Note that the path difference between the "long" and "short" paths is equivalent to $2 \theta$ : this relationship is valid for all values of $\theta$.

An explanation for the $p=0$ pulse at $\tau \approx 78$ fs is now needed. The $p=0$ ray in Fig. 7 is due only to external reflection from the sphere, whereas the Debye $p=0$ term also includes diffraction. As diffraction is typically explained as a wave phenomenon (in which the far field can be considered as the interference pattern due to, for example, a uniformly illuminated disk), diffraction cannot be represented by a single ray - unlike the other scattering mechanisms shown in Fig. 7 or Fig. 10.

Instead of just examining the impulse response at specific values of scattering angle $\theta$, much more information is revealed by studying how the impulse response varies with $\theta$, as shown in Fig. 11 (for the above listed conditions). In Fig. 11, the intensity of the scattered pulses is coded according to the false-color scale shown above the diagram. The maximum intensity occurs for $p=0$ at $\theta=0^{\circ}$ and $\tau \approx 67 \mathrm{fs}$. Note the time reference $(\tau=0)$ corresponds to reflection from the exterior of the sphere at $\theta=180^{\circ}$. 


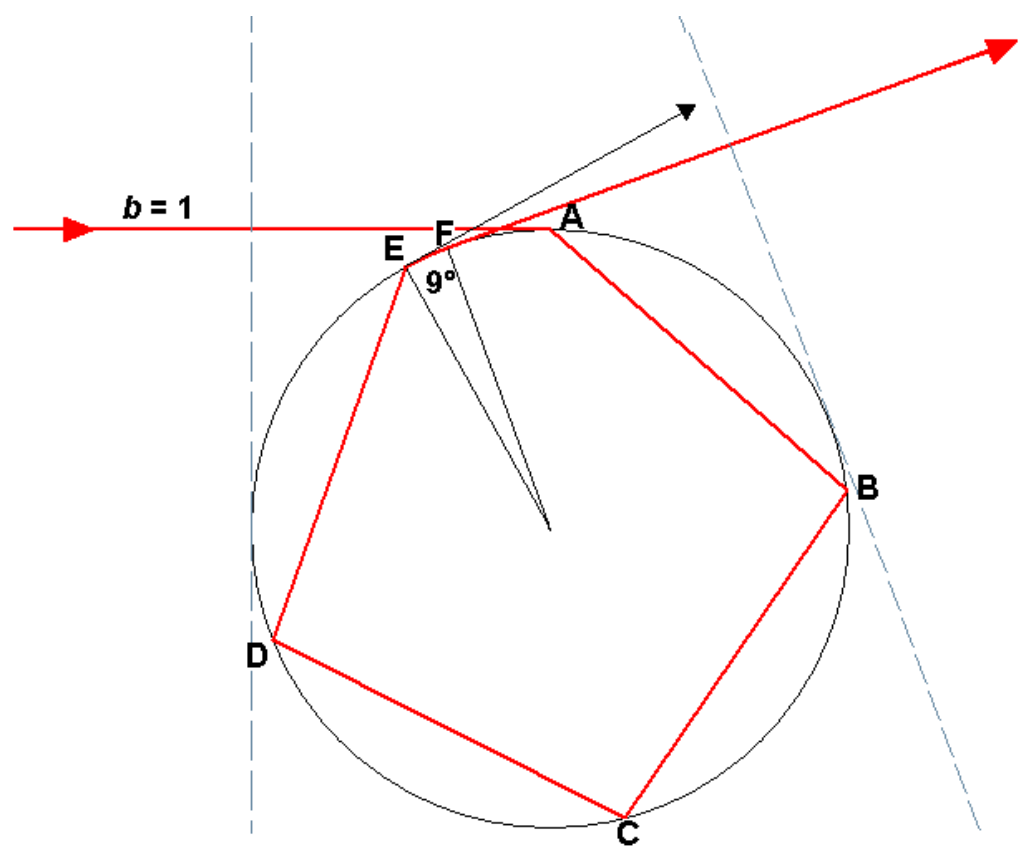

(a)

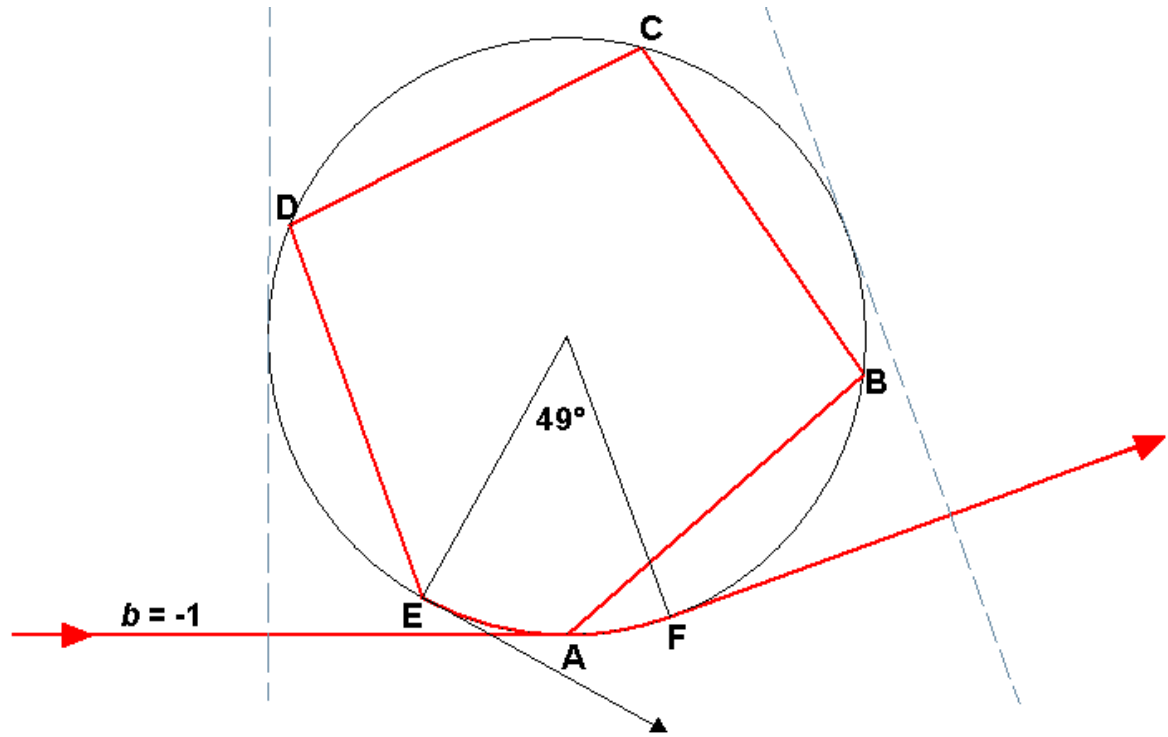

(b)

Fig. 10 . Scattering at $\theta=20^{\circ}$ caused by $p=4$ rays and surface waves for $n_{1}=1.3326$ :

a) impact parameter $b=1$ with a surface wave travelling $9^{\circ}$ clockwise;

b) impact parameter $b=-1$ with a surface wave travelling $49^{\circ}$ counterclockwise.

Note that, in both (a) and (b), the incident ray is tangential to the surface at point A, where it enters the sphere and then suffers internal reflections at B, C and D. Ray-tracing suggests that the $p=4$ ray would leave the sphere at point $E$ along the tangential black lines corresponding to $\theta=29^{\circ}$ in (a) and at $\theta=-$ $29^{\circ}$ in (b). However, surface waves can travel from $\mathrm{E}$ to $\mathrm{F}$ along the circumference of the sphere before following the tangential red lines at $\mathrm{F}$ at the desired value of $\theta=20^{\circ}$. 
Fig. 12 shows the results of Debye series calculations for $p=0$ and $p=1$ scattering. It also refers to ray-tracing calculations for reflection from the exterior of the sphere $(p=0)$ : the latter results are shown as a parametric curve plotting scattering angle $\theta$ against time delay $\tau$ for various values of the impact parameter $b$ :

- when $p=0$ and $b=0$ (i.e. a central ray), $\theta=180^{\circ}$ and $\tau=0$;

- when $p=0$ and $b=1$ (i.e. an edge ray), $\theta=0^{\circ}$ and $\tau=d\left(n_{0} / \mathrm{c}\right)$, where $d$ is the diameter of the sphere, $\mathrm{c}$ is the speed of light in vacuo and $n_{0}$ is the refractive index of the medium. In this particular case, $\tau=d\left(n_{0} / \mathrm{c}\right) \approx 66.67 \mathrm{fs}$.

The pulses due to $p=1$ scattering (i.e. transmission through the sphere) are delayed relative to the $p=0$ pulses: when $\theta=0^{\circ}$, the $p=1$ pulse occurs at $\tau=d\left(n_{1} / \mathrm{c}\right)$ where $n_{l}$ is the refractive index of the sphere: in this case, $\tau=d\left(n_{1} / \mathrm{c}\right) \approx 88.84 \mathrm{fs}$.

Figs. 11 and 12 demonstrate that it is possible to retrieve the intensities of the various scattering mechanisms by direct measurement of the impulse response at a given scattering angle. This goes even further than the mathematical approach of the Debye series because scattering for a given value of $p$ often involves a mixture of several ray paths (each of which results in a distinctive pattern on diagrams such as Fig. 11). For example, the complicated pattern of the $p=2$ curves in Fig. 11 can be used to identify the contributions of geometrical rays and surface waves.

Fig. 13 shows the results of ray-tracing calculations for $p=0$. In this case, the only scattering mechanism considered is that of reflection from the exterior of the sphere. This plot is comparable to the left part of the $p=0$ curve in Fig. 12. Despite their similarity, these two curves are slightly different when $\theta$ approaches $0^{\circ}$. To investigate these differences, the Fraunhofer approximation Eq. (1), has been used to produce Fig. 14, which surprisingly shows that "diffraction" produces a pattern that is symmetrical around $\tau=66.67 \mathrm{fs}$. The approximate nature of Eq. (1) is highlighted by the fact that the value of $\tau$ seems to reach an inflection point at $\theta=90^{\circ}$, whereas calculations using the Debye series for $\mathrm{p}=0$ indicate a linear relationship between $\tau$ and $\theta$ even when $\theta>90^{\circ}$. In any event, the Fraunhofer approximation is unlikely to be valid when $\theta>90^{\circ}$.

Fig. 13 indicates that the first $p=0$ pulse is due to reflection from the exterior of the sphere, but what about the second $p=0$ pulse highlighted by the dashed line in Fig. 12? I suggest that the second $p=0$ pulse is exclusively the result of diffraction. As this second $p=$ 0 pulse can be isolated by accepting only signals with $66.67 \mathrm{fs}<\tau<88.84 \mathrm{fs}$, I contend that it is possible to separate the diffracted component of the scattered signal.

The results in Fig. 14 indicate that the $p=0$ results shown in Fig. 10 for $\tau>66.67$ fs are, indeed, due to diffraction. However, Fig. 14 also shows that some of the $p=0$ results shown in Fig. 12 for $\tau<66.67$ fs are not solely due to reflection from the exterior of the sphere (because the impulse response for $p=0$ scattering in this region represents a mixture of diffraction and external reflection).

This mixture of scattering mechanisms disqualifies my attempt to claim the million-dollar prize. Even so, given the symmetry around $\tau=66.67 \mathrm{fs}$ in Fig. 14, it would be possible to determine indirectly the intensity of the total diffracted signal, which corresponds to double that of the diffracted signal measured in the range $\tau>66.67 \mathrm{fs}$.

Interestingly, if the diffraction calculation is repeated using a $50 \mathrm{fs}$ pulse (rather than the 5 fs pulse used in the above graphs), a very different pattern emerges, as shown in Fig. 15. Note that Fig. 15 covers a limited range of scattering angles $\left(0^{\circ}-30^{\circ}\right)$ rather than the $0^{\circ}-180^{\circ}$ range of the previous figures - thus showing that the resulting diffraction pattern has maxima and minima identical to those shown for diffraction in Fig. 5.

The impulse response techniques described in this section are, in principle, applicable to any size of sphere: instead of using $5 \mathrm{fs}$ pulses with spheres of $20 \mu \mathrm{m}$ diameter, $50 \mathrm{fs}$ pulses would give similar results with $200 \mu \mathrm{m}$ diameter spheres. However, $200 \mathrm{~nm}$ diameter spheres would require pulses of $0.05 \mathrm{fs}=50$ attoseconds duration - which would be very challenging! 


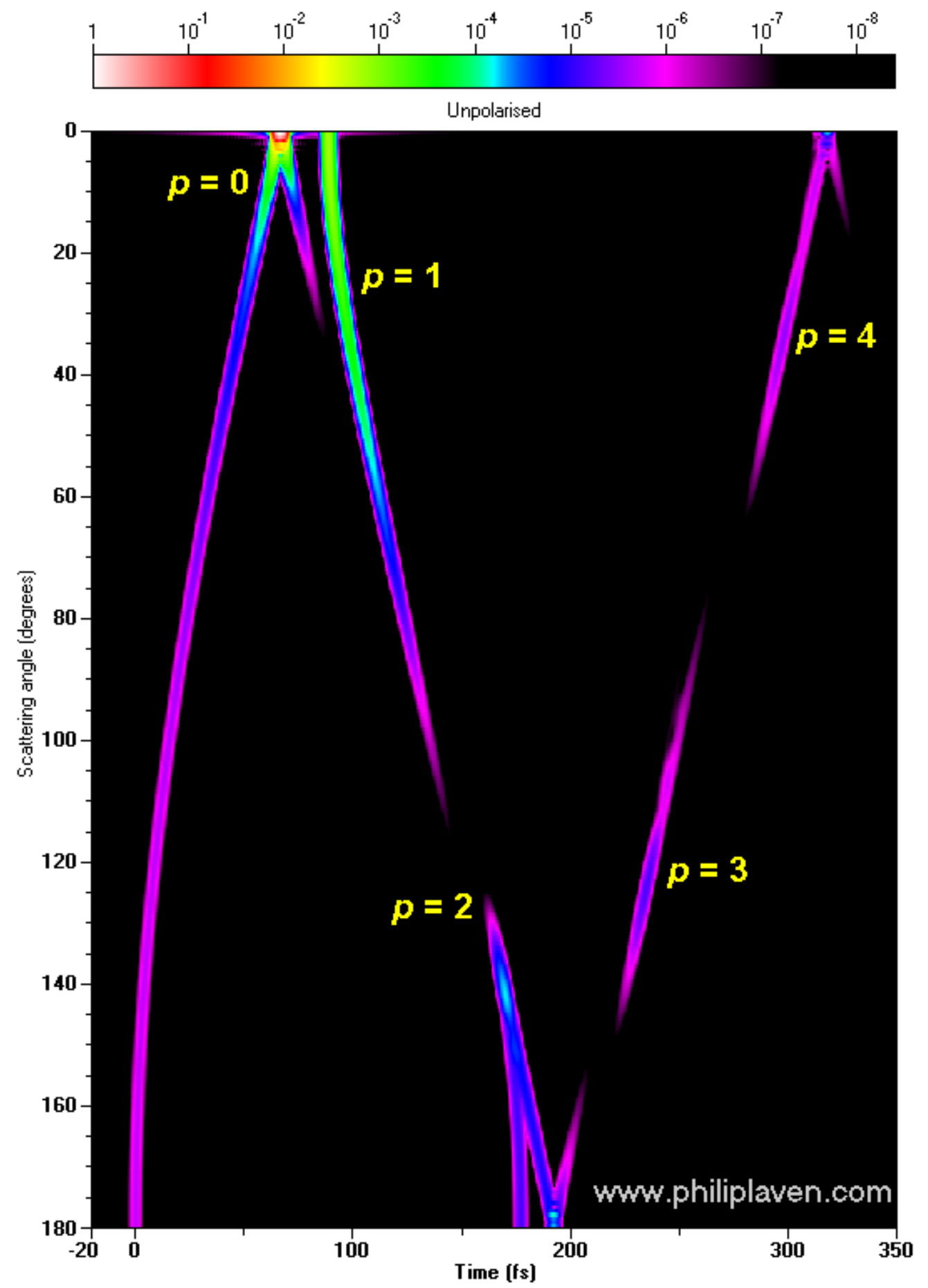

Fig. 11. Impulse response as a function of scattering angle for scattering of red light from a water droplet of diameter $d=20 \mu \mathrm{m}$. 


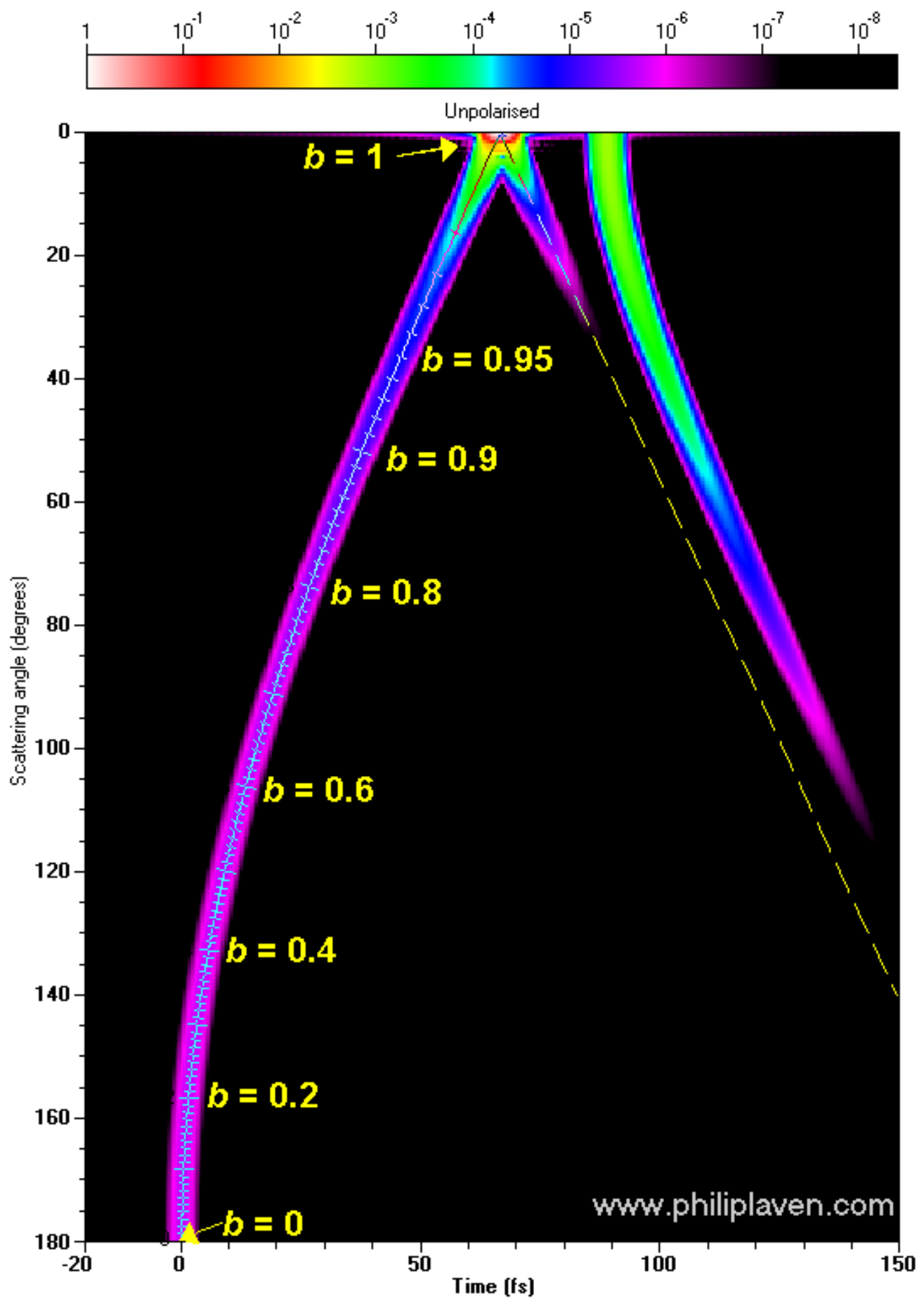

Fig. 12. Enlarged portion of Fig. 11 showing only $p=0$ and $p=1$ scattering. 


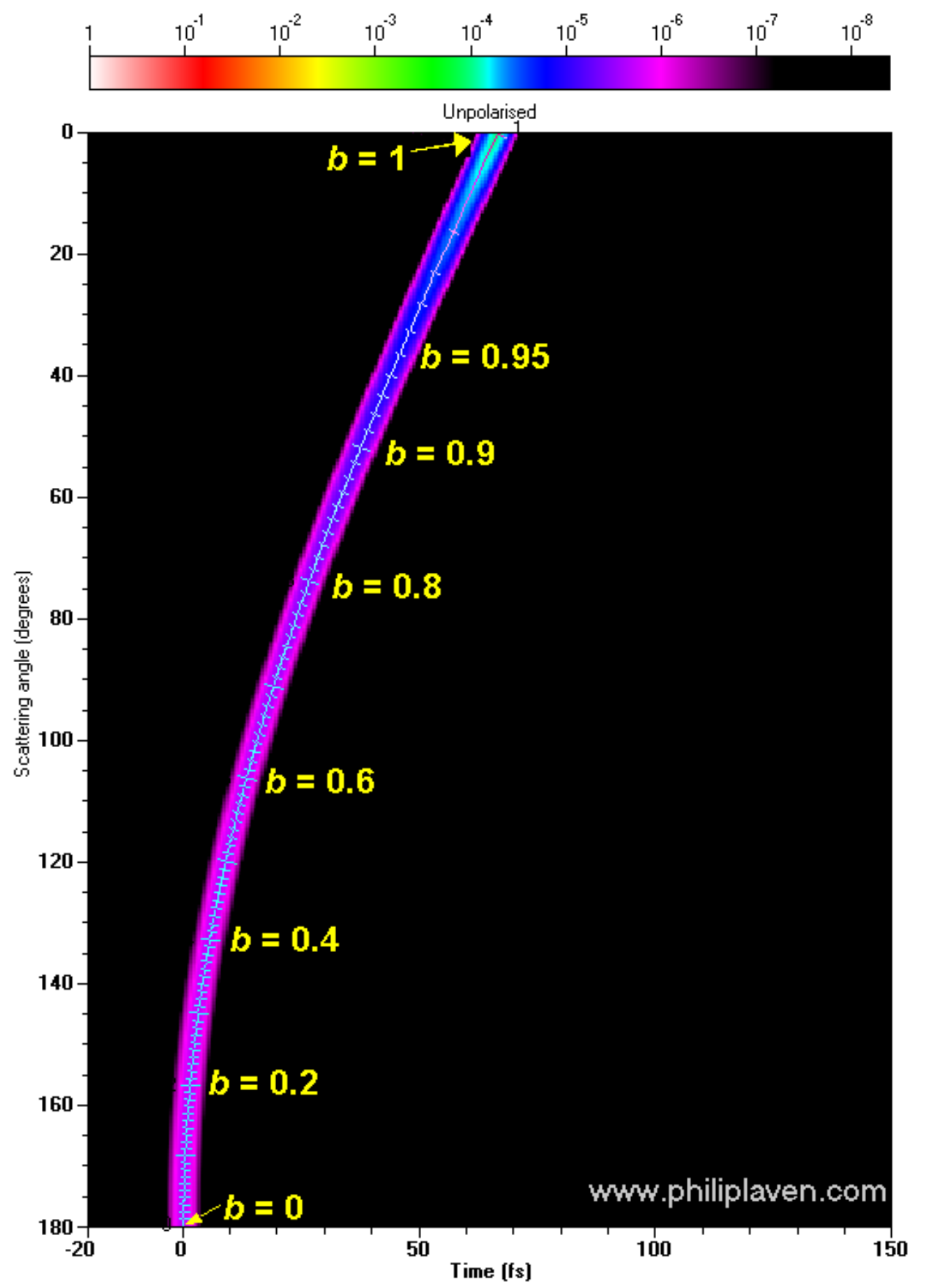

Fig. 13. As Fig. 12 but showing only the results for ray-tracing for $p=0$ (corresponding to reflection from the exterior of the sphere). 


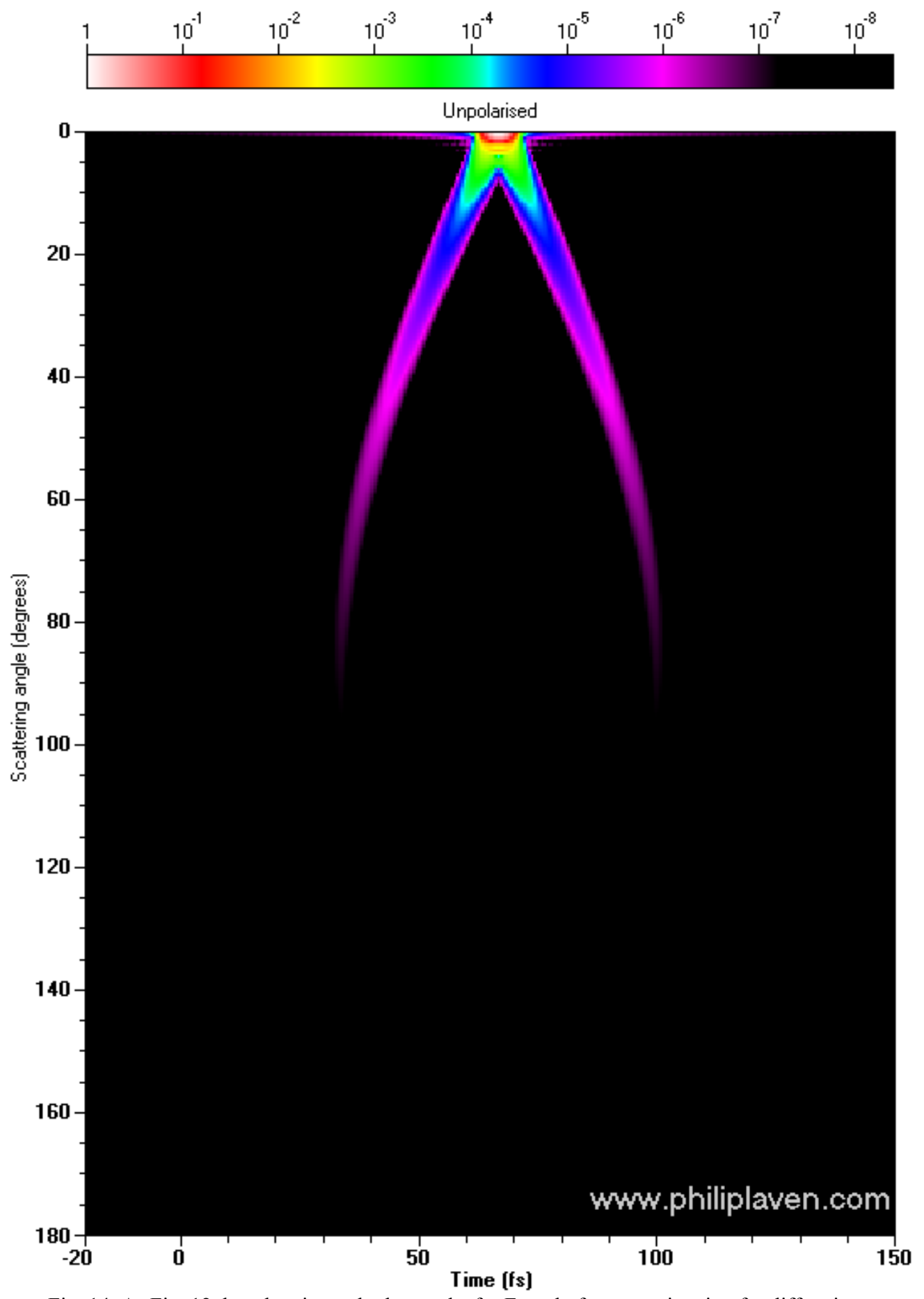

Fig. 14. As Fig. 12, but showing only the results for Fraunhofer approximation for diffraction. 


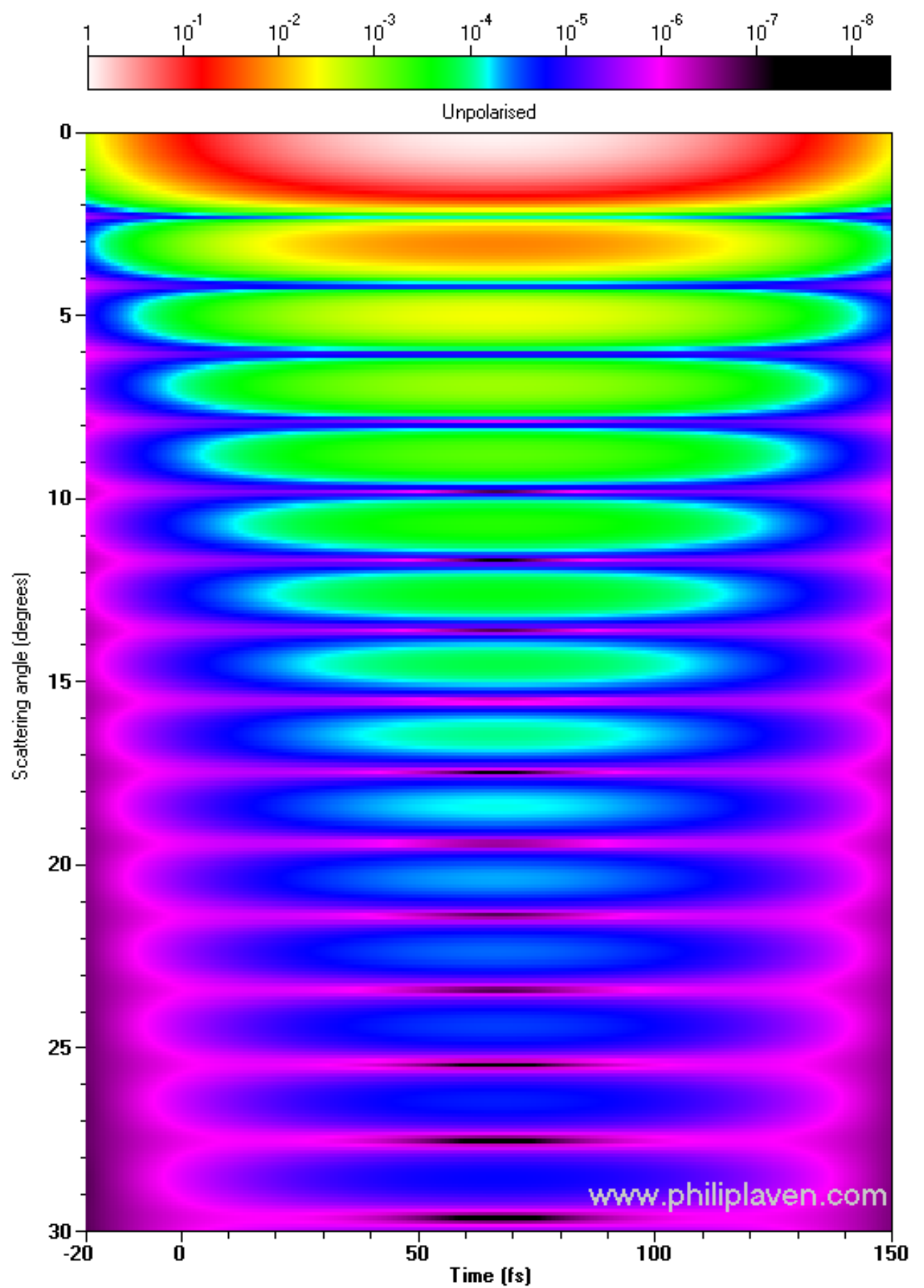

Fig. 15. Fraunhofer approximation for diffraction (as in Fig. 14 but with a pulse of $50 \mathrm{fs}$ duration). 


\section{CONCLUSIONS}

The million-dollar challenge set by Craig Bohren involves devising "a detector that distinguishes between scattered and diffracted waves, accepting the one but rejecting the other." This paper has analyzed this challenge from a theoretical perspective, using the example of scattering of red light from a spherical water droplet of diameter $d=20 \mu \mathrm{m}$. Although calculations using the Debye series can separate the contributions made by scattering of order $p$, Debye series calculations for $p=0$ combine diffraction with reflection from the exterior of the sphere. Nevertheless, the results show that measurements in the time domain (i.e. by measuring the impulse response due to the scattering of extremely short pulses of light) can distinguish between various scattering mechanisms, such as diffraction, transmission, reflections and surface waves. Unfortunately, these calculations also show that, for forward scattering, the time delays due to reflection from the exterior of the sphere are almost identical to the time delays for part of the diffracted signal. Consequently, as it does not seem possible to achieve complete separation of the diffracted signals (even with a pulse duration as short as $5 \mathrm{fs}$ ), this paper must record my failure to satisfy the challenge.

Despite this failure, the paper suggests that the intricacies of scattering can be revealed by considering the time domain (i.e. scattering of short pulses of light), instead of the usual assumption of a constant source of light. Measurements of the impulse response of a small sphere might be difficult to achieve in practice, but computer programs can produce detailed simulations of such measurements - thus achieving results that, in some ways, are more ambitious than Bohren's million-dollar challenge.

I am sure that Craig Bohren never expected serious attempts to solve his challenge - but, like most of us, he had not given sufficient consideration to the time domain concept of scattering. Even so, his million-dollar prize seems safe for the time being!

\section{APPENDIX: THE DEBYE SERIES}

Although the title of Debye's 1908 paper [3] refers to the "theory of the rainbow", it was primarily concerned with scattering from a cylinder. Debye did point out that "the extension to spheres is possible in an analogous way without difficulty" but this extension does not seem to have been addressed until 1937 when van der Pol and Bremmer tackled the problem. [4].

The amplitude of the scattered field at scattering angle $\theta$ is given by LMT as:

$$
\begin{aligned}
\mathrm{S}_{1}(\theta) & =\sum_{\mathrm{n}=1}^{\infty} \frac{2 \mathrm{n}+1}{\mathrm{n}(\mathrm{n}+1)}\left[\mathrm{a}_{\mathrm{n}} \pi_{\mathrm{n}}(\theta)+\mathrm{b}_{\mathrm{n}} \pi_{\mathrm{n}}(\theta)\right] \\
\mathrm{S}_{2}(\theta) & =\sum_{\mathrm{n}=1}^{\infty} \frac{2 \mathrm{n}+1}{\mathrm{n}(\mathrm{n}+1)}\left[\mathrm{a}_{\mathrm{n}} \tau_{\mathrm{n}}(\theta)+\mathrm{b}_{\mathrm{n}} \pi_{\mathrm{n}}(\theta)\right]
\end{aligned}
$$

In the Debye series expansion, the values of $a_{n}$ and $b_{n}$ are re-defined as:

$$
\begin{aligned}
& \mathrm{a}_{\mathrm{n}}=\frac{1}{2}\left[1-\mathrm{R}_{\mathrm{n}, \mathrm{TM}^{22}}-\sum_{p=1}^{\infty} \mathrm{T}_{\left.\mathrm{n}, \mathrm{TM}^{21}\left(\mathrm{R}_{\mathrm{n}, \mathrm{TM}}{ }^{11}\right)^{p-1} \mathrm{~T}_{\mathrm{n}, \mathrm{TM}}{ }^{12}\right]}\right. \\
& \mathrm{b}_{\mathrm{n}}=\frac{1}{2}\left[1-\mathrm{R}_{\mathrm{n}, \mathrm{TE}}{ }^{22}-\sum_{p=1}^{\infty} \mathrm{T}_{\mathrm{n}, \mathrm{TE}}{ }^{21}\left(\mathrm{R}_{\mathrm{n}, \mathrm{TE}}{ }^{11}\right)^{p-1} \mathrm{~T}_{\mathrm{n}, \mathrm{TE}^{12}}\right]
\end{aligned}
$$


The terms $\mathrm{R}_{\mathrm{n}}{ }^{22}$ and $\mathrm{R}_{\mathrm{n}}{ }^{11}$ are the partial-wave reflection coefficients, whilst $\mathrm{T}_{\mathrm{n}}{ }^{22}$ and $\mathrm{T}_{\mathrm{n}}{ }^{11}$ are the partial-wave transmission coefficients. The subscripts TE and TM indicate the polarization. The terms inside the square brackets in Eqs. (A.3) and (A.4) have specific physical meanings: the first term represents diffraction; the second term represents reflection from the exterior of the sphere and the third term represents $(p-1)$ internal reflections. If $\mathrm{a}_{\mathrm{n}}$ and $b_{n}$ are evaluated for a given value of $p$, Eqs. (A.1) and (A.2) can be used to calculate the amplitude of the scattered field $\mathrm{S}_{1, p}(\theta)$ and $\mathrm{S}_{2, p}(\theta)$ due to order $p$.

As noted in Section 2.1, the Debye series is not an approximation since the sum of the Debye series for all integer values of $p$ from $p=0$ to $p=\infty$ gives the same result as LMT calculations. In practice, this vector sum can be safely truncated at much lower values of $p$, for example, $p_{\max }=12$ (i.e. $p=0$ through $p=12$ ) is more than sufficient for scattering of red light from water droplets of diameter $d=20 \mu \mathrm{m}$. However, the number of terms necessary to achieve agreement with LMT results increases dramatically as $d$ is reduced below $1 \mu \mathrm{m}$ : for example, $p_{\max }=10^{4}$ is required when $d=0.2 \mu \mathrm{m}$, whereas $p_{\max }=10^{7}$ is required when $d=$ $0.02 \mu \mathrm{m}$. The extraordinary values of $p_{\max }$ required when $d<<1 \mu \mathrm{m}$ demonstrate that using the Debye series in these circumstances is like using a sledge-hammer to crack a nut. Of course, for scattering of light from such small particles, Rayleigh scattering is applicable which is much simpler than LMT or the Debye series!

It should be emphasized that the extremely slow convergence of the Debye series is not a problem in practice since it is generally only necessary to sum the Debye series contributions to check the accuracy of computer algorithms.

\section{Acknowledgements}

I would to thank the two anonymous reviewers for their invaluable and constructive suggestions which have helped to improve this paper.

\section{References}

[1] C. F. Bohren and E. E. Clothiaux, Fundamentals of Atmospheric Radiation, WileyVCH, Weinheim (2006). [doi: 10.1002/9783527618620]

[2] C. F. Bohren and D. R. Huffman, Absorption and Scattering of Light by Small Particles, Wiley-VCH, New York (1983). [doi: 10.1002/9783527618156]

[3] P. Debye "Das elektromagnetische Feld um einen Zylinder und die Theorie des Regenbogens," Physikalische Zeitschrift 9, 775-778 (1908) [N.B. An English translation of this paper entitled "The electromagnetic field around a cylinder and the theory of the rainbow" is available in Selected Papers on Geometrical Aspects of Scattering, SPIE Milestone Series Volume MS 89 (1993)]

[4] B. Van der Pol and H. Bremmer, "The diffraction of electromagnetic waves from an electrical point source round a finitely conducting sphere, with applications to radiotelegraphy and the theory of the rainbow," Philos. Mag. 24, 141-176, 825-864 (1937).

[5] E. A. Hovenac and J. A. Lock, "Assessing the contributions of surface waves and complex rays to far-field Mie scattering by use of the Debye series," J. Opt. Soc. Am. A 9, 781-795 (1992) [doi:10.1364/JOSAA.9.000781]

[6] H. C. van de Hulst, Light Scattering by Small Particles, Dover, New York (1981) (reprint of 1957 Wiley edition) [doi:10.1063/1.3060205]

[7] M. Born and E. Wolf, Principles of Optics: Electromagnetic Theory of Propagation, Interference and Diffraction of Light ( $7^{\text {th }}$ Edition), Cambridge University Press, Cambridge (2000) [doi:10.2277/0521642221]

[8] H. Bech and A. Leder, "Particle sizing by ultrashort laser pulses - numerical simulation," Optik 115, 205-217 (2004) [doi:10.1078/0030-4026-00361] 
[9] H. Bech and A. Leder, "Particle sizing by time-resolved Mie calculations - A numerical study," Optik 117, 40-47 (2006) [doi:10.1016/j.ijleo.2005.06.008]

Philip Laven retired in 2007 as Technical Director of the European Broadcasting Union, based in Geneva, Switzerland. Previously, he worked for the BBC in London where he held various senior posts, such as Controller of Engineering Policy from 1992 to 1997 and Chief Engineer (R\&D) from 1989 to 1992 . He is currently Chairman of DVB (www.dvb.org) which develops standards for digital TV. 